\title{
Adaptive System for of Load Capacity in Piles Based on Error Minimization
}

\author{
Alcineide Pessoa ${ }^{1}$, Carolina Rosa ${ }^{2}$, Raphael Magalhães ${ }^{3}$, Gean Sousa ${ }^{4}$, Luiz Mauricio Furtado Maués ${ }^{5}$ \\ ${ }^{1}$ Institute of Technology, Federal University of Pará, Belém, Brazil \\ ${ }^{2}$ University of Cuiabá \\ ${ }^{3}$ Institute of Technology, Federal University of Pará, Belém, Brazil \\ ${ }^{4}$ Department of Science and Technology, Federal University of Maranhão, São Luís-MA , Brazil \\ ${ }^{5}$ Institute of Technology, Federal University of Pará, Belém, Brazil
}

Correspondence Author: Alcineide Pessoa, Institute of Technology, Federal University of Pará, Belém, Brazil

E-mail: - alcineidedutra@hotmail.com

Received date: 25 July 2019, Accepted date: 28 September 2019, Online date: 25 October 2019

Copyright: $\odot 2019$ Alcineide Pessoa, et al, This is an open-access article distributed under the terms of the Creative Commons Attribution License, which permits unrestricted use, distribution, and reproduction in any medium, provided the original author and source are credited.

\begin{abstract}
Calculating load capacity is a major problem in geotechnics. The solution of this problem through computational mechanisms has instigated several researchers. In this sense, this article aims to present a computational model capable of calculating the load capacity from information such as length, diameter and type of pile. The methodology used is to model a system that has as theoretical basis the working principles of adaptive filters. In the construction of the model and validation, a total of $\mathbf{3 0 6}$ compressive load tests were used. To verify the efficiency of the proposed methodology, the cross-validation method was used. It has been found in computer simulations that the adaptive algorithm is capable of predicting load capacity with good accuracy. The best result obtained had a Root Mean Square Error -RMSE of $518.99 \mathrm{KN}$ and a Pearson correlation coefficient of $0.91 \%$.
\end{abstract}

Keywords: Load Capacity, Adaptive Filters, Cross Validation, Root Mean Square Error

\section{INTRODUCTION}

Civil engineering comprehends several areas and, in each area,, several challenges are found. The solutions to these challenges are supported on mathematical, computational and empirical mechanisms. In this context, an area that stands out is Geotechnics, in particular, the study of foundations.

The geotechnical study of foundations, specially soil-pile interaction has been an important challenge, even more, when the task is to predict the load capacity in piles. This task is characterized by the difficult mathematical or computational modeling of the physical problem. Due to these difficulties, several theoretical and semi-empirical models have been studied (Odebrecht, 2003; Aoki and Cintra, 2000).

The theoretical approaches that focus on determining the load capacity are numerous, the classic works of Terzaghi (1943), Meyerhof (1951), Berezantev (1961) and Vésic (1972) are highlighted. Among the semi-empirical models, the most utilized are the method of Aoki \& Velloso (1975), that of Décourt \& Quaresma (1978) and Antunes e Cabral (1996).

Recently, computational models were used in order to enhance the quality of load capacity estimates. Prayogo and Susanto (2018) presented a methodology based on artificial intelligence to predict load capacity. Other authors such as Erman et al. (2015), Zhang and Goh (2016), Moayedi and Armaghani (2018) and Rashid et al. (2019) also used computational models capable of calculating the rupture load on piles.

Despite the diversity of classical and computational models, there is still a lack of generalization of the presented models, since each one applies to a restricted reality of a given region, considering the details of the soil of the studied spot. Another weakness of the existing models is the low applicability in the engineer's daily life, given the complexity of the proposed algorithms.

To minimize the error between values resulting from a computational model and those obtained in situ, this paper proposes a computationally adaptive system with an easy implementation that aims to predict the load capacity in different types of piles. The variables used in the model are easy to obtain and can be adapted to different situations, guaranteeing a generalization of the 
proposed solution. It is noteworthy that the calculation of the load capacity through a computer system is the main objective of this work and that such system is provided in the methodology and its effectiveness presented in the obtained results.

\section{RUPTURE LOAD AND ADAPTIVE SYSTEMS}

The main contribution of this work is to use an adaptive system to calculate load capacity on piles. The concepts and examples of these two topics (adaptive system and load capacity) are summarized below.

\subsection{Load Capacity}

An isolated pile admissible load is, by definition, the force applied on the pile that causes settlement compatible with the construction, considering satisfactory safety against ground and foundation rupture. Load-capacity verification mechanisms are mainly focused on two large groups: rational (or theoretical) methods and semi-empirical methods.

The theoretical methods are based on variables such as friction angle and soil cohesion. On the other hand, the semi-empirical methods statistically relate the load capacity of the element with in situ tests (Terzaghi, 1943). It is usual to assume that the load capacity comes from the balance between the applied load and the soil resistance. Mathematically, this static equilibrium can be expressed by equation 1

$Q_{U}=A_{p} \cdot q_{p}+U \cdot \sum_{i=1}^{n} \tau_{i} \Delta L$

Where:

$A_{p}=$ Tip or pile base area;

$q_{p}=$ Tip unitary resistance;

$U=$ Pile perimeter;

$\tau_{i=\text { side unitary resistance; }}$

$\Delta L=$ the length of the pile to which $\tau_{l}$ applies.

Based on this equilibrium principle, three methods stand out: Aoki-Velloso (1975), Décourt-Quaresma (1978) and Antunes and Cabral (1996). The Aoki-Velloso method (1975) is a proposal that is strongly correlated to laboratory tests. The mathematical model established in this method is given by the following expression:

$Q_{U}=\frac{k N_{p}}{F_{1}} \cdot A_{p}+\frac{U}{F_{2}} \cdot \sum_{L=1}^{n} \alpha \cdot k \cdot N_{L} \cdot \Delta L$

where $\mathrm{k}$ is a constant characteristic of the soil type, $N_{p}$ is the value of $N_{\text {spt }}$ at the tip support, $\alpha$ the coefficient dependent of the soil type, and $N_{L}$ is the mean value of $N_{s p t}$ referring to $\Delta \mathrm{L}, F_{1}$ e $F_{2}$ are correction factors that depend on the type of pile, according to table 1 .

Table 1: Constants F1 and F2 proposed by Aoki \& Veloso (1975).

\begin{tabular}{|c|c|c|}
\hline Type of Pile & $F 1$ & $F 2$ \\
\hline Franki & 2,50 & 5,00 \\
\hline Metalic & 1,75 & 3,50 \\
\hline Digged & 3,50 & 7,00 \\
\hline Root & 2,00 & 4,00 \\
\hline Continuos Helix & 2,00 & 4,00 \\
\hline Omega & 2,00 & 4,00 \\
\hline
\end{tabular}

Décourt-Quaresma (1978) proposed a method in which the pile type is dependent on two parameters $\alpha$ and $\beta$. The mathematical expression presented to calculate the load capacity is:

$Q_{U}=\alpha . C . N_{p} A_{p}+\beta .10 .\left(\frac{N_{L}}{3}+1\right) U . L$ 
where $\mathrm{C}$ is a constant characteristic of the soil, $\mathrm{L}$ the height of the soil layer and the other variables have already been defined previously.

Using a set of load tests applied to helical-type piles Antunes and Cabral (1996) presented the mathematical model (Equation 4) to predict load capacity.

$$
Q_{U}=U \cdot \sum\left(N . \beta_{1}\right) \Delta L+\beta_{2} \cdot N \cdot A_{p}
$$

where $\beta_{1}$ and $\beta_{2}$ are soil characteristics dependent constants, $\mathrm{N}$ is an index of penetration resistance and the other variables have already been defined.

\subsection{Adaptive Systems}

According to Boroujeny (2013), an adaptive system is one that adjusts in response to information from its surroundings, modifying its parameters in order to achieve a well-defined goal. These systems can be linear or not. Haykin (2003) highlights that adaptive systems are used to remodel a given input so that its output is a good estimate of the desired values. The process of selecting the system parameters is done through the optimization of a properly defined performance function (Bertsekas, 2012).

The most commonly used performance function is the Mean Square Error between the desired signal and the system output (Boroujeny, 2013). When the computational algorithm reaches the optimal value of the cost function, it is said that the system has adapted its parameters. Figure 1 is a diagram representing the general scheme of an adaptive system.

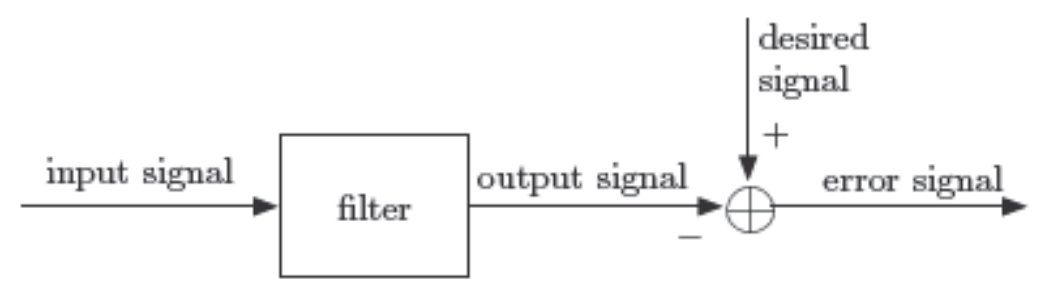

Figure 1: Generic scheme of an adaptive system (Boroujeny, 2013).

Although the system presented in Figure 1 seems simple, several challenges and applications are inherent to these types of systems. Challenges such as analyzing filter parameters and/or creating cost functions that are not error-based. Among the applications of this class of systems, filtering of signals and the prediction of numerical series is one of the most important.

Adaptive systems are widely used in applications such as noise-cancelling, time series prediction, prediction of time series, etc. According to Widrow (1985) the basis of adaptive systems is the Least Mean Squares (LMS) algorithm.

From the LMS several algorithms were derived, including some models of Artificial Neural Networks (Haykin, 2008). The LMS algorithm is quite simple and the update of its parameters is given by:

$W_{l k+1}=W_{l k}+2 \mu . \varepsilon_{k} \cdot x_{k N}$

where $\mathrm{W}$ is a coefficient vector, $x_{k}$ is the k-esimal entry, $\mu$ is a convergence factor and $\varepsilon_{k}$ is the difference between the output and the desired signal (or measured values). The system output signal consists of the linear combination between the inputs and the updated coefficients.

\section{MATERIALS AND METHODS}

\subsection{Database}

The database used in the computational experiments performed is composed of 306 compressive load tests. These samples were obtained from a compilation of works by Lobo (2005). Figure 3 shows the percentage of data that compose the base by the Brazilian state. The variables of the database used were: type, length and diameter of the pile. 


\section{Distribution of the Database by Brazilian State}

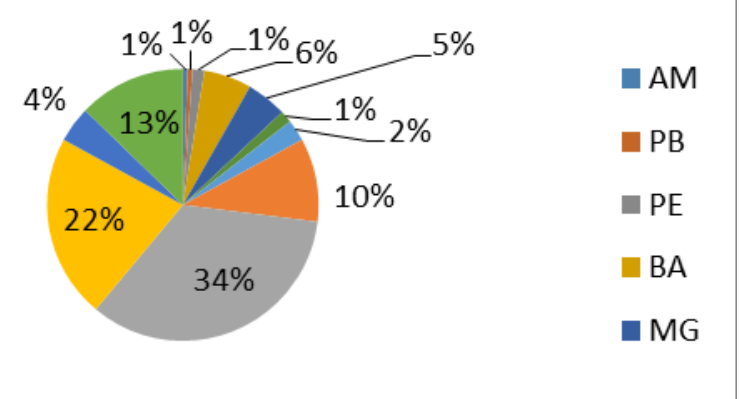

Figure 3: Composition of the database in percentage of load tests by Brazilian state: AM- Amazonas, PB-Paraíba, PEPernambuco, BA-Bahia, MG-Minas Gerais, DF-Distrito Federal, ES- Espírito Santo e RJ-Rio de janeiro.

Figure 3 shows that the database used is quite heterogeneous, and this fact indicates a possible generalization of the results found.

\subsection{Proposed estimation system}

The proposed adaptive system to perform estimation of rupture load on piles is based on the solution of an optimization problem whose objective function is the error. The system plan is shown in figure 4.

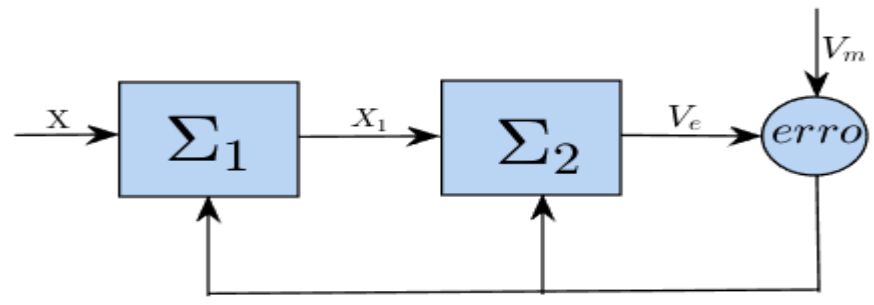

Figure 4 - Plant of the proposed system

The system parameters are $X$ - input vector; $\sum_{1}=A X ; X_{1}=f(A X)+B$; the $f$ function is non-linear (Ex. hyperbolic tangent, sigmoid, etc.); $\sum_{2}=A_{1} X_{1} ; V_{e}=f\left(A_{1} X_{1}\right)+B_{1}$ (method's estimated value); $V_{m}$-measured value and erro $=V_{m}-V_{e}$.

Given that $X$ and $V_{m}$ are known, it remains to establish an algorithm capable of estimating the matrixes $A, A_{1}, B$ and $B_{1}$, such that the "erro" is minimal. In this paper, only the matrixes $A$ and $A_{1}$ are estimated. To $B$ and $B_{1}$ fixed values are established. Estimating the matrixes $A$ and $A_{1}$ so that the error is minimal is an unrestricted optimization problem that is widely addressed in the literature and one of the mathematical methods used is the Gradient Descent Method (Bertsekas, 2012). According to Bazarra (2006), the Gradient Descent Method the matrix $A$ that minimizes the error can be found iteratively through the following algorithm:

$$
A_{\text {atual }}=A_{\text {anterior }}+\alpha \cdot \nabla \text { erro, }
$$

where $\alpha$ is a constant that determines the speed with which the algorithm achieves the minimum error and $\nabla$ erro is the error gradient? An analogous procedure is used to estimate the value of $A_{1}$. It is worth mentioning that the error is a function of $A$ and $A_{1}$, so when calculated the gradient, it will also have the same size of the matrix under analysis and so the sum will be possible.

The algorithm for updating the matrices then acts iteratively until it reaches the minimum error, and when this occurs it is said that the system has adapted to the problem and can be tested in new samples already using the matrices found in the adaptation phase.

\subsection{Crossed Validation}

In order to verify the efficiency of adaptive systems used in prediction and or classification, it is recommended to use the method known as "k-fold cross-validation". According to Varoquaux (2018), this method consists of dividing the data set into k 
subsets of approximately equal size. The $\mathrm{k}-1$ partition objects are used in the parameter estimation process and validation is performed using the remaining partition. This process is repeated $\mathrm{k}$ times. The performance is then evaluated from the mean of the evaluation metrics (error and correlation). This technique can be illustrated from figure 5.

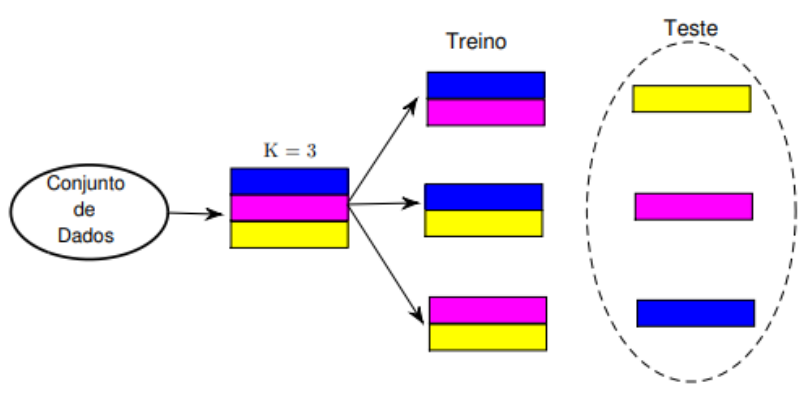

Figure 5: Cross Validation method

\subsection{Statistical Methods}

The metrics used for performance evaluation of the proposed system are Root Mean Square Error -RMSE (Pham et al., 2018)

error $=\sqrt{\frac{\sum_{i=1}^{n}\left(y_{i}-d_{i}\right)^{2}}{n}}$

and the Pearson correlation coeficient (Rodriguez, 2010):

$$
\text { correlation }=\frac{\operatorname{cov}(y, d)}{\sqrt{\operatorname{var}(y) x \operatorname{var}(d)}}
$$
values.

where $\operatorname{cov}(y, d)$ is the covariance, $\operatorname{var}(y)$ is the variance of the system output values and the variance of the measured

\section{RESULTS}

The computational simulations were performed in two stages. The first step was to adapt the parameters of the system, in this phase 276 samples of the data set were used. The second stage consisted of the validation of the system already adapted to the problem under analysis. For the validation, 30 samples were used. The input variables in the system were F1, F2, length and diameter of the pile, in addition to $B$ and $B_{1}$ that were fixed as vectors whose elements are numbers 1 . The value of $\alpha$ in all the simulations was 0.9. The software used in the simulations was Matlab.

Samples used both in the adaptation phase and in the validation phase were randomly chosen. In the adaptation phase, the cross-validation technique with 10 folds $(\mathrm{k}=10)$ was used. The mean error obtained in the 10 folds in the validation was 964.67 with a standard deviation of 243.04 . The mean correlation was $90.4 \%$ with a deviation of $1.17 \%$.

Figure 6 is a comparison between the actual values and those obtained by the system in the adaptation phase considering the best result obtained (error $=1312$ and correlation $=92 \%$ ). 


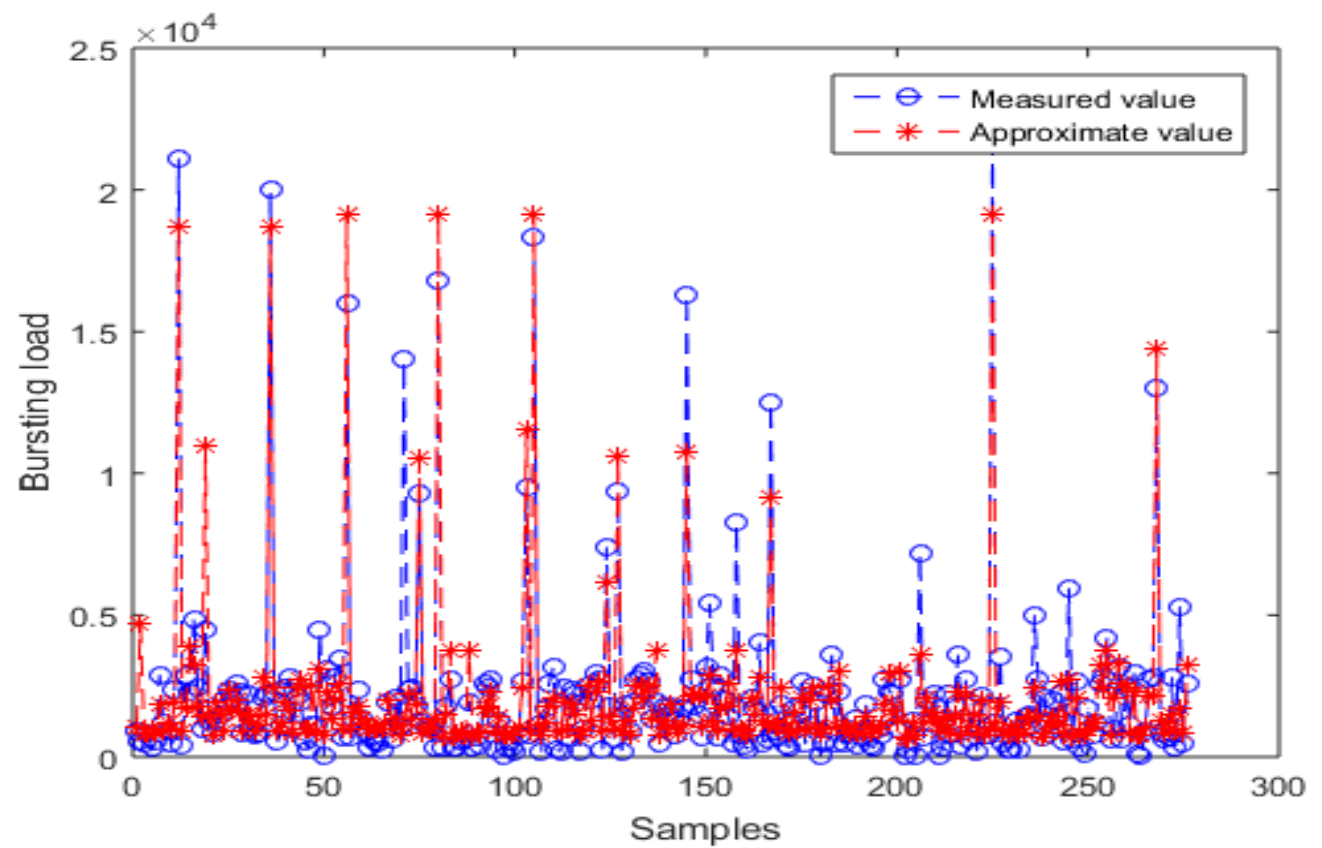

Figure 6: Comparison between the actual values and those obtained by the proposed system in the adaptation phase.

It can be seen in Figure 6 that there is an approximation between the values obtained by the system and the measured samples. In addition, the error and correlation values at this stage were also quite satisfactory. These facts indicate the possibility of obtaining good results in the test phase.

The optimal matrixes obtained in the adaptation of the system were:

$$
A=\left[\begin{array}{cccc}
0.88 & 1.89 & 0.77 & -0.08 \\
1.12 & 1.28 & 0.70 & -0.18 \\
1.25 & 1.16 & 0.39 & -0.09 \\
1.02 & 1.18 & 0.65 & 0.23
\end{array}\right] \text { and } A_{1}=\left[\begin{array}{llll}
2.22 & 1.51 & 0.66 & 0.72
\end{array}\right]
$$

Matrixes $A$ and $A_{1}$ are extremely important in the use of this methodology, since the main objective in the adaptation phase is to find them. Using these matrices found in the adaptation phase of the system was validated. Figure 7 consists of a graphical comparison between the actual values and those obtained by the system in the validation phase for the best result obtained. This figure shows, graphically, that there was a good adherence of the estimated data to the measured values. In the best result the error (RSME) was 518.99.

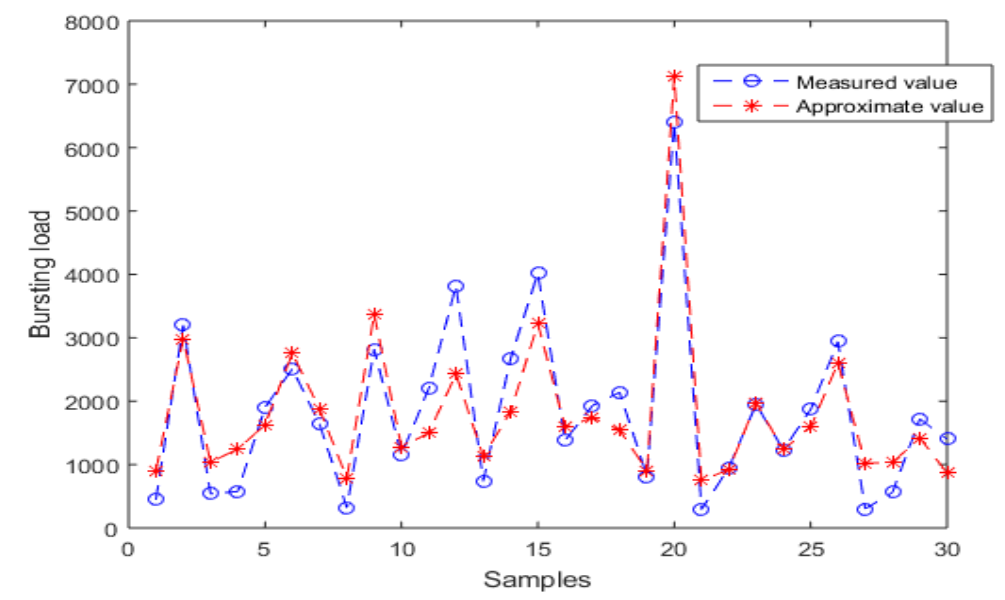

Figure 7: Comparison between the actual values and those obtained by the proposed system in the validation phase. 
Citation: Alcineide Pessoa, et al., Adaptive System for of Load Capacity in Piles Based on Error Minimization. Australian Journal of Basic and Applied Sciences, 13(10): 25-32. DOI: $10.22587 /$ ajbas.2019.13.10.4

The dispersion of the points in relation to approximate values $\mathrm{x}$ measured values can be observed in figure 8 . This figure illustrates how the data approaches a line $(y=0.99 x+4.8)$ indicating a good correlation between them. In this specific case the correlation coefficient was equal to 0.916 .

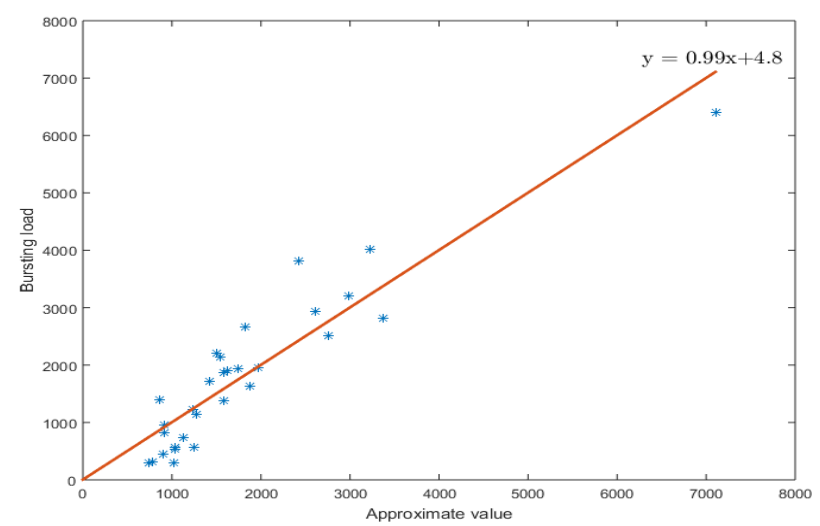

Figure 8: Correlation between the actual values and those obtained by the proposed system in the validation phase.

\section{DISCUSSION AND CONCLUSION}

The adaptive computational system proposed in this work was adapted to the problem in which it was inserted, since it converged to minimize the error and to optimize the correlation between the measured values and those obtained by the system. Through cross-validation one can observe a stability in the adaptation process, since the error values in each fold were not absurdly discrepant (deviation $=243.04)$.

The matrixes resulting from the adaptation process are not high dimensional, which provides an application of the model in other databases. The error in the validation phase was relatively small (518.99) and this may indicate an applicability of the model, since the correlation between the system output and the actual values was high $(91.68 \%)$.

The methodology proposed in this article differs from the classical methodologies mainly because it has an inherent power of generalization of the adaptive models. In addition, it is a computationally simple methodology, and this fact is an advantage when compared to the existing artificial intelligence-based computational models, especially neuronal models.

It is worth mentioning that the proposed system requires only three information (length, diameter and type of pile) to calculate load capacity, while most models require a lot of information. Another advantage of this type of system is that it can be implemented in future computer applications and made available for daily use.

The combination of characteristics such as stability in the adaptation process, generalization of the solution of the problem, small error and high correlation in the validation phase, simplicity of computational implementation and practical applicability of the system, indicate that the proposed methodology is presented as advancement in relation to the calculation load capacity on piles.

\section{REFERENCES}

Antunes, W.R. and D. A Cabral, 1996. Capacidade de carga em estacas hélice contínua. $3^{\circ}$ Seminário de Engenharia de Fundações e Geotecnia. São Paulo, 2: 105 - 109.

Aoki, N. and D. A. Veloso, 1975. An Approximate Method to Estimate the Bearing Capacity of Piles. In: Panamerican Conference on Soil Mechanics and Foundation Engineering (PCSMFE). Buenos Aires.

Aoki, N. and J.C.A. Cintra, 2000. The application of energy conservation Hamilton's principle to the determination of energy efficiency in SPT tests. Proceedings the Sixth International Conference on the Application of Stress-Wave Theory to Piles, 457-460.

Bazaraa, M.S., H. D. Sherali and C. M. Shetty, 2006. Nonlinear programming:Theory and algorithms, 3rd ed. John Wiley \& Sons.

Berezantsev, V. G. Khisttoforov and V. S. Golubkov, 1961. Load bearing capacity and deformation of pile foundations. In: $5^{\circ}$ CONGRÈS INTERNATIONAL DE MÉCANIQUE DES SOLS ET DES TRAVAUX DE FOUNDATION, Paris.

Bertsekas, D. P., 2015 Convex optimization algorithms. Athena Scientific, 2015.

Boroujeny, B. F., 2013. Adaptive filters: theory and applications, John Wiley \& Sons.

Décourt, L. and A. R. Quaresma, 1978. Capacidade de carga de estacas a partir de valores de SPT. In: $6^{\circ}$ CONGRESSO BRASILEIRO DE MECÂNICA DOS SOLOS E ENGENHARIA DE FUNDAÇÕES - CBMSEF, Rio de Janeiro.

Erman, O., F. Milad, T. Kamal, H. Nader, 2015. New method for predicting the ultimate bearing capacity of driven piles by using Flap number, KSCE Journal of Civil Engineering,3:611-620.

Haykin, S. and B. Widrow, 2003. Least-Mean-Square Adaptive Filters, Wiley-Interscience, Hoboken, NJ.

Lobo, B. O., 2005. Métodos de Previsão de Capacidade de Carga de Estacas: Aplicação dos Conceitos de Energia do Ensaio SPT. 2005. Porto Alegre.

Meyerhor, G. G., 1951. The ultimate bearing capacity of foundations. Geotechnique, vol 2. 
Moayedi, H. and D. J. Armaghani, 2018. Optimizing an ANN model with ICA for estimating bearing capacity of driven pile in cohesionless soil. Engineering with Computers, 2: 347-356.

Odebrecht, E., 2003. Medidas de energia no ensaio SPT, Tese de Doutorado, Universidade federal do Rio Grande do Sul, Porto Alegre.

Pham B. T., L. H. Son, T. Hoang, D. Nguyen and D. T. Bui, 2018. Prediction of shear strength of soft soil using machine learning methods. Catena.1:181-191.

Prayogo, D. and Y. Susanto, 2018. Optimizing the Prediction Accuracy of Friction Capacity of Driven Piles in Cohesive Soil Using a Novel Self-Tuning Least Squares Support Vector Machine. Advances in Civil Engineering, 1 (2):121-131.

Rashid, A., H. Moayedi, M. Raftari, A. Sharifi, W. A. W. Jusoh, 2019. Optimization of ANFIS with GA and PSO estimating $\alpha$ ratio in driven piles. Engineering with Computers, 1:1-12.

Rodriguez L., R. Huerta, C. Elkan and Cruz, 2010. Seleção de característica de programação quadrática J. Mach. Aprender.

Terzagui, K., 1943. Theoretical soil mechanics. New York.

Varoquaux, C., 2018. Cross-validation failure: Small sample sizes lead to large error bars. NeuroImage 1:68-77.

Vésic, A. S., 1972. Expansion of cavities in infinite soil mass. Journal of the Soil Mechanics and Foundations Division, ASCE, Vol 98: 3.

Widrow, Bernard, Stearns and D. Samuel, 1985. Adaptive Signal Processing (1st ed.). Prentice-Hall.

Zhang W. and A. t. C. Goh , 2016. Multivariate adaptive regression splines and neural network models for prediction of pile drivability. Geoscience Frontiers, 1: 45-52. 